Orthopäde 2014 - 43:501-502

DOI 10.1007/s00132-014-2291-5

Online publiziert: 4. Juni 2014

(c) Springer-Verlag Berlin Heidelberg 2014

\author{
W. Mittelmeier \\ Orthopädische Klinik und Poliklinik, Universitätsmedizin Rostock
}

\title{
Qualitätssicherung in der Endoprothetik
}

Sehr geehrte Leserinnen und Leser,

die Qualität der Patientenversorgung und die Sicherheit von Implantaten stehen immer wieder in der öffentlichen Kritik. Dieser Band von Der Orthopäde beleuchtet Kernfragen dieser Thematik.

Die moderne Endoprothetik leistet einen ganz entscheidenden, zunehmenden Beitrag zur Aufrechterhaltung eines sehr hohen selbständigen Mobilitätsgrads unserer älter werdenden Gesellschaft mit sehr hohen Erfolgsquoten. Im Rahmen der Einführung neuerer Implantate treten aber immer wieder Versagensfälle auf.

Die Erwartungen an Implantate und an die handelnden Personen steigen in unserer Gesellschaft stetig. Die Anforderungen präklinischer Prüfungen und $\mathrm{Zu}$ lassungsverfahren wie auch die Vergabe des CE-Kennzeichens sind umstritten, insbesondere die Zulassung nach Baugleichheit („substantial equivalence certification") in Analogie zu einem bestehenden Implantatsystem.

Die Prüfungen nach ISO-Normen unterliegen einerseits hohen Anforderungen, beschreiben aber als Mindestvoraussetzungen letztlich nur einen Teil der tatsächlichen Belastung in vivo. Sie entstehen unter aufwendiger internationaler schrittweiser Einigung von Expertengruppen der herstellenden Industrie. Der zunehmende Kostendruck seitens der Hersteller wirkt einer ständigen Veränderung und Verschärfung der Normen entgegen.

Die neueren Technologien präoperativer Simulationen über Finite-Elemente- und Hardware-in-the-loop-Techniken bieten sich zunehmend für Worstcase-Simulationen im Sinne höherer Implantatsicherheit an.
Letztendlich stellen die verwendeten Werkstoffe in Abhängigkeit von Design und Beanspruchung die wesentliche Grundlage sicherer Implantate dar. Der Einsatz geeigneter Werkstoffe unter Berücksichtigung der spezifischen Belastungsgrenzen ist unabdingbar für Hersteller und Anwender im Hinblick auf nachhaltige Versorgungsqualität. Große Variabilität des Polyethylens einerseits und seine Belastungsgrenzen als einem der grundlegenden Werkstoffe der Endoprothetik werden hier aufgezeigt.

Ein zentrales Qualitätsprofil besteht in der Lernkurve des Operationsteams. Dieses ist durch verschiedene Parameter von Patient und Implantatsystem geprägt, aber auch wesentlich durch Schulung, Erfahrung und Training. Eine erhöhte Operationsdauer kann dabei ein messbarer Parameter für das Qualitätsmanagement sein. Der Umgang mit Lehreingriffen ist ebenso verantwortungsvoll wie das rechtzeitige Erkennen von Qualitätsproblemen im Team.

In den letzten Jahren zeigt sich ein verstärktes Engagement der Fachgesellschaft DGOOC und der Arbeitsgemeinschaft für Endoprothetik hinsichtlich qualitätsverbessernder Maßnahmen, aber auch der Einsatz namhafter Hersteller für das Prinzip des „no train - no use“. In der Erprobungsphase von EndoCert bereits konnten in allen Kliniken eine wesentlich bessere Struktur- und Prozessqualität verzeichnet werden.

In der Versorgung von Komplikationen wie periprothetischen Frakturen zeigen sich technische Grenzen durch interindividuell unterschiedliche Voraussetzungen einschließlich der begrenzten Verankerungsmöglichkeiten und reduzierter Knochenqualität. 
Der zunehmende Leistungsdruck durch verpflichtende Register und konsequente Zertifizierungsverfahren wie die EndoCert-Initiative (der Fachgesellschaft DGOOC mit AE und BVOU) werden die Sicherheit und Leistungsfähigkeit der Endoprothetik weiter erhöhen.

Die Leistungsfähigkeit zur Detektion der Standzeit verschiedener Endoprothesenfabrikate hat in verschiedenen Registern in den letzten Jahren überzeugt. Möglichkeiten und Grenzen der Register zur frühen Entdeckung möglicher Versagensserien werden kritisch beleuchtet.

Die qualitative Bedeutung computerassistierter Instrumentationen wie der Knieendoprothesennavigation bedarf nach der Euphoriephase der letzten Jahre einer kritischen Analyse.

Sachgerechte Aufbewahrung und systematische Schadensanalyse von Explantaten im Falle unerwartet frühen Implantatversagens sind wesentliche Voraussetzung für frühzeitiges und reproduzierbares Lernen aus diesen Fällen. Dies gilt nicht nur für Implantatkombinationen, sondern auch für schadhafte Instrumente.

Letztlich steht auch die Endoprothetik unter dem Druck der begrenzten Ressourcen des Gesundheitssystems, sodass auch ein - zumindest kurzfristiger - Vergleich dieser operativen Maßnahmen mit der konservativen Therapie in diesem Themenheft nicht gescheut werden darf.

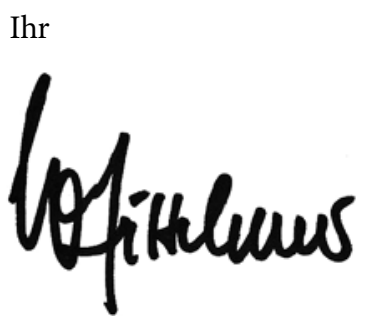

Prof. Dr. Wolfram Mittelmeier

\section{Korrespondenzadresse}

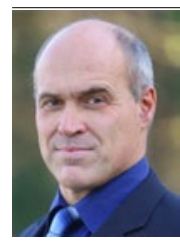

Prof. Dr. W. Mittelmeier

Orthopädische Klinik und Poliklinik, Universitätsmedizin Rostock

Doberaner Straße 142,

18057 Rostock

wolfram.mittelmeier@

med.uni-rostock.de

\section{Einhaltung ethischer Richtlinien}

Interessenkonflikt. W. Mittelmeier gibt an, dass kein Interessenkonflikt besteht.

\section{Wissenschaftspreis 2014 der AE - Deutsche Gesellschaft für Endoprothetik}

Die AE-Deutsche Gesellschaft für Endoprothetik schreibt den Wissenschaftspreis 2014 als Auszeichnung für eine innovative wissenschaftliche Arbeit auf dem Gebiet der Endoprothetik aus.

Teilnahmeberechtigt sind Orthopäden und Unfallchirurgen und alle Mitglieder der $\mathrm{AE}$ und $\mathrm{AE}-\mathrm{ComGen}$. Eingereicht werden können ausschließlich Arbeiten, die in einem peer reviewed Journal publiziert oder zur Publikation angenommen worden sind. Die Publikation darf nicht älter als ein Jahr sein. Es werden nur Arbeiten berücksichtigt, die nicht gleichzeitig für einen anderen Preis eingereicht oder bereits anderweitig ausgezeichnet worden sind.

Die Dotierung beträgt EUR 10.000,--

Die Arbeiten müssen in deutscher oder englischer Sprache abgefasst sein und in 6- facher Ausfertigung bis zum 20.09.2014 bei der Geschäftsstelle der AE-Deutsche Gesellschaft für Endoprothetik e.V., Oltmannsstraße 5, 79100 Freiburg, eingegangen sein.

Die eingereichten Arbeiten werden von einer unabhängigen Jury bewertet.

Beim 16. AE-Kongress am 05./06. Dezember 2014 in Bonn sollte die/der Preisträger/ in in einem Referat einen Überblick über die Arbeit geben.

Der Rechtsweg ist ausgeschlossen.

Weitere Informationen:

Tel. 0761 / 4564 - 7666, www.ae-germany.com 